\title{
Collaborative Online Learning in Undergraduate Medical Education: A Scoping Review
}

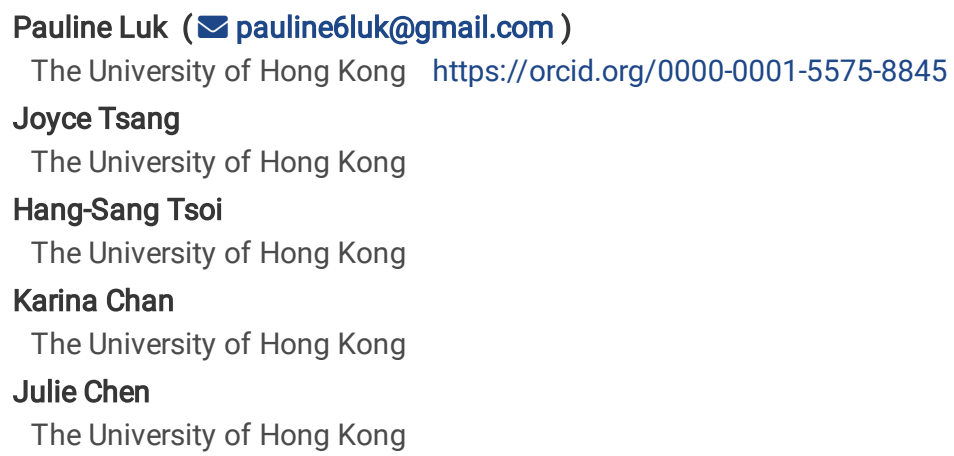

Research article

Keywords: Collaborative Online Learning, Undergraduate, Medical Education

Posted Date: December 7th, 2020

DOI: https://doi.org/10.21203/rs.3.rs-28397/v2

License: (c) (i) This work is licensed under a Creative Commons Attribution 4.0 International License. Read Full License 


\section{Abstract}

Background:

Collaborative online learning is an active learning method that stimulates interaction between learners and teachers and fosters interest and motivation in learning in a virtual learning environment.

Aim:

The primary aim of this scoping review is to examine the extent and nature of academic publications on collaborative online learning in undergraduate medical education.

Methods:

We used a standardized framework for conducting scoping reviews to identify suitable publications and to analyze the content.

Results

Thirty-six published articles from January 1990-March 2019 met the inclusion criteria. There were 16 interventional studies, 8 reviews, 7 commentaries and 5 survey studies. Most work originated in the UK or the US. These studies found that collaborative online learning demonstrated educational value by personalizing learning to fit individual needs, promoting social interaction and active learning, and helping to build professional networks Connectivity, ease of use, and accessibility were enablers of collaborative online learning while technical challenges were the main barrier.

\section{Conclusion}

There is a relatively small but promising body of work currently published on collaborative online learning in undergraduate medical education. Further work and innovation in this area may be forthcoming in response to the need for alternatives to traditional learning in times of crisis.

\section{Background}

\section{What is collaborative online learning?}

Collaborative online learning is an active learning method that stimulates interaction between learners and teachers and foster interest and motivation in learning in a virtual learning environment (1). Since the advent and popularization of the internet in the 1990s, the growing influence of digital and social media has reshaped the teaching and learning ecology in medical education. Online learning has been an increasingly popular means to engage learners and teachers in medical education $(2,3)$ particularly as students have access to computer technology in learning, through personal smartphones, laptop computers, and other wireless mobile devices. (4) Collaborative online learning in medical education uses a variety of tools for teaching and learning such as blogs $(5,6)$, Facebook $(7,8)$, Google Sites $(6,9)$, Moodle $(10,11)$, Twitter $(7,12)$, etc. all of which have shown encouraging educational outcomes.

\section{Why is it collaborative online learning important?}

Numerous studies have shown that collaborative online learning can supplement conventional teaching practices and provide a more engaging and effective learning environment for teachers and learners $(2,13-15)$.

The interactive nature of collaborative online learning encourages active learning and enhances the learning experience by connecting and allowing dynamic creation of knowledge for students in individual and collective levels (16). Students can attain a higher degree of engagement in an online and can engage in team discussions and share their experiences with peers (4). Collaborative learning allows students to feel that they are part of a supportive community where they can share relevant knowledge, give effective feedback, ask for help and support through difficult stages, draw encouragement from other facing similar issues, and work constructively towards a common goal $(11,17)$.

Online collaborative learning improves learning effectiveness (14), cost $(2,18)$, and promotes efficient use of time $(4,19)$. It has been associated with higher academic achievement, deeper levels of learning, and retention of learned information for longer times (11). Online collaborative learning can also train students to be self-motivated (4) and help students to acquire learning skills such as, better problem solving and higher-order critical thinking skills (11). The online platforms are effective for time management and facilitate schedule constraints by providing flexibility for students to work on their own time and pace (4).

Collaborative online learning allows learners to co-create learning materials that suit their needs and learning styles. There is also evidence that collaborative online learning in medical education accommodates different learning styles of learners, is responsive to learner needs, and is effective in changing the learning outcomes of learners (19). These outcomes relevant to medical education include competencies like professionalism $(5,15,20)$, and interprofessional collaboration $(2,21)$.

Page $2 / 21$ 


\section{Why do we need to have a scoping review on this topic?}

Online collaborative learning is an opportunity for medical education to take advantage of the increasing availability and potential for mobile and technology-driven approaches (6). The application, advantages, and challenges of using collaborative online learning in medical education are currently under-explored (22) and it is important to identify factors that will promote collaborative online learning and foster the effectiveness of this mode of learning in a technologically advanced and information-rich world (13). It is also necessary to develop and implement novel teaching and learning strategies to boost collaborative online learning in medical education (16).

\section{What do we want to explore?}

This scoping review aims to examine the extent and nature of research articles published on collaborative online learning in the medical education literature. This will help to inform research directions and pedagogical applications for collaborative online learning in medical education in the future.

\section{Methods}

A scoping review is a subtype of systematic review aiming to map the available research studies related to the key concepts underpinning a specific topic area with time (when it was published), location (country), source (peer-reviewed or grey literature), and or origin (healthcare or academic discipline) (23). It aims to understand the influence of such work and the depth and breadth of the field, to identify gaps in the research studies, and report on the types of research studies that address and inform practice (23). It enables the clarification of complex areas of inquiry and refines and expands the essential concepts on the areas of subsequent research studies (2). This is often a preliminary part of an effectiveness review and leads to refined search strategies etc. (24).

We followed the BEME Guide No. 3 systematic searching for evidence in medical education (24) and Arksey and O’Malley's five-step framework for interpreting scoping literature reviews (25) to conduct this scoping review.

\section{Stage 1: Identifying the Research Questions}

To explore the literature on collaborative online learning in medical education, the following research questions were generated:

- What is the extent and nature of the academic publications on collaborative online learning in medical education?

- What insights about collaborative online learning have we learned from the literature?

\section{Stage 2: Identifying Relevant Studies}

Guided by the research questions and following a consultation with the university medical librarian, we searched for articles using MeSH terms in PubMed and expand the search to relevant terms in other databases. These key terms "collaborat*", "online learning" (MeSH), "eLearning", "internet", "Education, Medical, Undergraduate" (MeSH), "medical education”, "undergraduate”. We searched these terms in seven electronic databases: PubMed, ERIC, JSTOR, ProQuest (including Australian Education Index, Education, Collection, Medical Database, and PsycINFO) for articles from 1990 to March 2019.

We undertook an additional search of online publications through Google and Google Scholar, and a further hand search of the reference list of all selected articles.

\section{Stage 3: Study Selection}

The inclusion criteria for this review were: 1) academic publications published in English; 2) relating to one or more of the three key concepts: "collaborative", "online learning", "medical education", and/or "undergraduate program" 3) involving medical students as the subjects or topic.

We excluded papers based on the following exclusion criteria: 1) solely non-medical education, (e.g. those solely focused on nursing, dentistry, occupation therapy, pharmacy, physiotherapy, etc.) and 2) solely non-undergraduate level of studies (e.g. those solely focusing on postgraduate education, continuing medical education (CME), distance learning with unrestricted participants, etc.).

After the first screening of title and abstract by the first reviewer, 183 articles were selected for a full review. Four study team members (PL, JT, HT, $\mathrm{KC}$ ) conducted the reviews. Each article was screened independently by two reviewers to determine if the article fulfilled the inclusion and exclusion criteria. Where there was ambiguity, three team members (PL, JT, HT) reviewed the article together in full, and resolved differences by consensus. Articles that consisted of abstracts or brief discussions were excluded. A flow chart of the article screening process is shown in Figure 1.

Of the 36 articles, 17 related to all four key areas: "collaborative", "online learning", "medical education”, and/or "undergraduate program". Four papers did not specifically mention collaborative learning but discussed online learning in general including its collaborative features. Three papers did not specifically mention online learning but addressed it from a wider perspective. Two papers included mix of students from different 
disciplines including medical students. Ten papers involved undergraduate education to some extent, and eight papers did not specify the level of education.

\section{Stage 4: Charting the Data}

Key information was extracted by categorizing the nature of the selected articles. The following information was extracted from each of the included studies:

- study aims

- population, sample size

- types, period, location, and duration of the intervention

- methods /analysis

- reported outcomes

\section{Stage 5: Collating, Summarizing and Reporting the Results}

A thematic analysis was undertaken to identify the key issues addressed in each paper which were then subdivided into themes. Information was tabulated and collated to gain insight into the features of collaborative online learning in medical education.

\section{Results}

\section{The Extent and Nature of Research Studies}

\section{Characteristics of Selected Studies}

Of the 36 relevant papers that underwent a full review, we found that 16 of were empirical studies focused on implementation and evaluation of an education program/element, 5 studies reported survey results on the use of social media, 7 were systematic or scoping reviews, and 8 were expert perspectives on collaborative online learning in medical education. (Table 1)

The progressive prominence of personal computing and the Internet since the 1990's provided a favorable environment for collaborative learning (26). However, the earliest article related to collaborative online learning in medical education appeared in 2003 with a steady increase in publications since 2007. In each year, there was an average of 2 to 4 papers published on this topic. (Table 1) 


\begin{tabular}{|c|c|c|c|c|c|c|c|c|}
\hline Author & Year & Country & $\begin{array}{l}\text { Methods/ } \\
\text { Analysis }\end{array}$ & $\begin{array}{l}\text { Population } \\
\text { (course, year, } \\
\text { university) }\end{array}$ & $\begin{array}{l}\text { Sample } \\
\text { Size }\end{array}$ & Aim/Purpose of study & Conclusion & Focus \\
\hline \multicolumn{9}{|l|}{ Surveys } \\
\hline $\begin{array}{l}\text { Dommett, } \\
\text { E. J. }\end{array}$ & 2019 & UK & $\begin{array}{l}\text { Online } \\
\text { survey }\end{array}$ & $\begin{array}{l}\text { full-time } \\
\text { undergraduates, } \\
\text { King's College } \\
\text { London }\end{array}$ & 50 & $\begin{array}{l}\text { To investigate the } \\
\text { students' perception of } \\
\text { the use of online } \\
\text { forums and twitter in } \\
\text { learning, relating use } \\
\text { to learning outcomes } \\
\text { and rating the } \\
\text { importance of } \\
\text { different factors in } \\
\text { using each tool }\end{array}$ & $\begin{array}{l}\text { Twitter provides } \\
\text { access to news media } \\
\text { and those outside their } \\
\text { program whilst forums } \\
\text { support discussion } \\
\text { with peers. A key } \\
\text { factor was whether } \\
\text { students felt that use } \\
\text { of a tool would } \\
\text { increase their grade, } \\
\text { indicating that } \\
\text { assessment outcomes } \\
\text { are strongly linked to } \\
\text { use of both tools, } \\
\text { despite them differing } \\
\text { in terms of } \\
\text { interactions and } \\
\text { specific uses. }\end{array}$ & $\begin{array}{l}\text { Prevalence } \\
\text { of usage }\end{array}$ \\
\hline $\begin{array}{l}\text { Gray, K., } \\
\text { et al. }\end{array}$ & 2010 & Australia & $\begin{array}{l}\text { Paper } \\
\text { survey } \\
\text { and case } \\
\text { studies }\end{array}$ & $\begin{array}{l}\text { undergraduate } \\
\text { medical } \\
\text { students, a } \\
\text { large } \\
\text { metropolitan } \\
\text { university }\end{array}$ & $\begin{array}{l}759+4 \\
\text { Study } \\
\text { Groups }\end{array}$ & $\begin{array}{l}\text { To investigate the } \\
\text { extent to which } \\
\text { medical students were } \\
\text { using Facebook and to } \\
\text { detail the approaches, } \\
\text { experiences and } \\
\text { outcomes in cases } \\
\text { where they were using } \\
\text { Facebook to support } \\
\text { their learning and to } \\
\text { gain insights into the } \\
\text { benefits and } \\
\text { challenges of using } \\
\text { social networking } \\
\text { software for learning } \\
\text { and teaching in } \\
\text { medicine and in higher } \\
\text { education generally }\end{array}$ & $\begin{array}{l}\text { Using Facebook as } \\
\text { part of learning and } \\
\text { teaching is as much of } \\
\text { a challenge for many } \\
\text { students as it may be } \\
\text { for most educators. }\end{array}$ & $\begin{array}{l}\text { Prevalence } \\
\text { of usage, } \\
\text { Effectiveness }\end{array}$ \\
\hline $\begin{array}{l}\text { Lemley, T. } \\
\text { and J. } \\
\text { Burnham }\end{array}$ & 2009 & US & $\begin{array}{l}\text { Online } \\
\text { survey }\end{array}$ & $\begin{array}{l}\text { medical and } \\
\text { nursing } \\
\text { educators, (DR- } \\
\text { ED, AACN, } \\
\text { AAHSL) }\end{array}$ & 55 & $\begin{array}{l}\text { to investigate the } \\
\text { actual use of web } 2.0 \\
\text { tools in the medical } \\
\text { and nursing } \\
\text { curriculum in an effort } \\
\text { to ascertain whether or } \\
\text { not use of these tools } \\
\text { has increased, thereby } \\
\text { creating a potential } \\
\text { role for medical } \\
\text { librarians }\end{array}$ & $\begin{array}{l}\text { Web } 2.0 \text { tools } \\
\text { portends a growing } \\
\text { trend in education, } \\
\text { training for medical } \\
\text { students, faculty and } \\
\text { practitioners is a } \\
\text { golden opportunity. }\end{array}$ & $\begin{array}{l}\text { Educational } \\
\text { value, } \\
\text { Prevalence } \\
\text { of usage }\end{array}$ \\
\hline $\begin{array}{l}\text { Sandars, } \\
\text { J., et al. }\end{array}$ & 2008 & UK & $\begin{array}{l}\text { Paper } \\
\text { survey }\end{array}$ & $\begin{array}{l}1 \text { st year, } \\
\text { University of } \\
\text { Leeds }\end{array}$ & 212 & $\begin{array}{l}\text { To identify the nature } \\
\text { and extent of the use } \\
\text { of social software by } \\
\text { first year medical } \\
\text { students }\end{array}$ & $\begin{array}{l}\text { Medical educators } \\
\text { need to recognize the } \\
\text { potential of social } \\
\text { software in } \\
\text { undergraduate } \\
\text { medical education but } \\
\text { it is essential that } \\
\text { students maintain the } \\
\text { informality and } \\
\text { privacy of these sites. }\end{array}$ & $\begin{array}{l}\text { Prevalence } \\
\text { of usage }\end{array}$ \\
\hline $\begin{array}{l}\text { Sandars, } \\
\text { J. and S. } \\
\text { Schroter }\end{array}$ & 2007 & UK & $\begin{array}{l}\text { Online } \\
\text { survey }\end{array}$ & $\begin{array}{l}\text { medical } \\
\text { students }+ \\
\text { practitioners, } \\
\text { British Medical } \\
\text { Association }\end{array}$ & 593 & $\begin{array}{l}\text { To identify the current } \\
\text { familiarity and use of } \\
\text { Web } 2.0 \text { technologies } \\
\text { by medical students } \\
\text { and qualified medical } \\
\text { practitioners, and to } \\
\text { identify the barriers to } \\
\text { its use for medical } \\
\text { education }\end{array}$ & $\begin{array}{l}\text { The potential of Web } \\
2.0 \text { technologies for } \\
\text { undergraduate and } \\
\text { postgraduate medical } \\
\text { education will only be } \\
\text { achieved if there is } \\
\text { increased training in } \\
\text { how to use this new } \\
\text { approach. }\end{array}$ & $\begin{array}{l}\text { Prevalence } \\
\text { of usage, } \\
\text { Enablers and } \\
\text { barriers }\end{array}$ \\
\hline
\end{tabular}




\begin{tabular}{|c|c|c|c|c|c|c|c|c|}
\hline Author & Year & Country & $\begin{array}{l}\text { Methods/ } \\
\text { Analysis }\end{array}$ & $\begin{array}{l}\text { Population } \\
\text { (course, year, } \\
\text { university) }\end{array}$ & Sample Size & $\begin{array}{l}\text { Aim/Purpose of } \\
\text { study }\end{array}$ & Conclusion & Focus \\
\hline \multicolumn{9}{|c|}{ Interventional Studies } \\
\hline $\begin{array}{l}\text { Saqr, M., } \\
\text { et al. }\end{array}$ & 2018 & $\begin{array}{l}\text { Saudi } \\
\text { Arabia }\end{array}$ & $\begin{array}{l}\text { Social } \\
\text { network } \\
\text { analysis }\end{array}$ & $\begin{array}{l}\text { clinical } \\
\text { reasoning, } \\
\text { Qassim } \\
\text { University }\end{array}$ & 82 & $\begin{array}{l}\text { To investigate } \\
\text { how social } \\
\text { network analysis } \\
\text { (interactivity, role } \\
\text { in information } \\
\text { exchange and } \\
\text { role in } \\
\text { collaboration, } \\
\text { group cohesion) } \\
\text { can be used to } \\
\text { monitor online } \\
\text { collaborative } \\
\text { learning, find } \\
\text { aspects in need } \\
\text { of improvement, } \\
\text { guide an } \\
\text { informed } \\
\text { intervention, and } \\
\text { assess the } \\
\text { efficacy of } \\
\text { intervention } \\
\text { using an } \\
\text { experimental, } \\
\text { observational } \\
\text { repeated- } \\
\text { measurement } \\
\text { design in three } \\
\text { courses over a } \\
\text { full-term } \\
\text { duration }\end{array}$ & $\begin{array}{l}\text { The intervention } \\
\text { has significantly } \\
\text { enhanced } \\
\text { student-student } \\
\text { and teacher- } \\
\text { student } \\
\text { interactions. } \\
\text { SNA can be used } \\
\text { for monitoring } \\
\text { and supporting } \\
\text { teaching and } \\
\text { learning in } \\
\text { higher } \\
\text { education. }\end{array}$ & \\
\hline $\begin{array}{l}\text { Ho, A., et } \\
\text { al. }\end{array}$ & 2018 & Singapore & $\begin{array}{l}\text { Data } \\
\text { analytics }\end{array}$ & $\begin{array}{l}\text { 1st and 2nd } \\
\text { year, National } \\
\text { University of } \\
\text { Singapore }\end{array}$ & 300 & $\begin{array}{l}\text { To share a case } \\
\text { of using a closed } \\
\text { Facebook group } \\
\text { to disseminate } \\
\text { health-related } \\
\text { new articles, and } \\
\text { facilitate } \\
\text { discussions that } \\
\text { facilitate co- } \\
\text { learning of real- } \\
\text { life ethical } \\
\text { issues, by } \\
\text { analyzing } \\
\text { comments and } \\
\text { reactions }\end{array}$ & $\begin{array}{l}\text { Facebook group } \\
\text { did not require } \\
\text { extensive } \\
\text { curriculum } \\
\text { changes and } \\
\text { was an efficient } \\
\text { pedagogical } \\
\text { platform. }\end{array}$ & Effectiveness \\
\hline $\begin{array}{l}\text { Cole, D., } \\
\text { et al. }\end{array}$ & 2017 & UK & Survey & $\begin{array}{l}\text { case-based } \\
\text { learning, } 1 \text { st } \\
\text { year, Cardiff } \\
\text { University }\end{array}$ & 71 & $\begin{array}{l}\text { To explore how } \\
\text { university- } \\
\text { supported } \\
\text { curation } \\
\text { platform and } \\
\text { external social } \\
\text { media } \\
\text { (Facebook) } \\
\text { could support } \\
\text { collaborative } \\
\text { small group } \\
\text { working in case- } \\
\text { based learning, } \\
\text { by surveys, data } \\
\text { and group } \\
\text { activities }\end{array}$ & $\begin{array}{l}\text { Optimum use of } \\
\text { social media } \\
\text { depends on } \\
\text { sufficient } \\
\text { training of both } \\
\text { staff and } \\
\text { students, and an } \\
\text { opportunity to } \\
\text { practice using } \\
\text { them, with } \\
\text { ongoing support. } \\
\text { The platforms } \\
\text { can all support } \\
\text { collaborative } \\
\text { learning, and } \\
\text { may help } \\
\text { develop digital } \\
\text { literacy, critical } \\
\text { appraisal skills, } \\
\text { and awareness } \\
\text { of wider health } \\
\text { issues in society. }\end{array}$ & Effectiveness \\
\hline
\end{tabular}




\begin{tabular}{|c|c|c|c|c|c|c|c|c|}
\hline Author & Year & Country & $\begin{array}{l}\text { Methods/ } \\
\text { Analysis }\end{array}$ & $\begin{array}{l}\text { Population } \\
\text { (course, year, } \\
\text { university) }\end{array}$ & Sample Size & $\begin{array}{l}\text { Aim/Purpose of } \\
\text { study }\end{array}$ & Conclusion & Focus \\
\hline $\begin{array}{l}\text { Peacock, } \\
\text { J. G. and } \\
\text { J. P. } \\
\text { Grande }\end{array}$ & 2016 & US & Survey & $\begin{array}{l}\text { pathology, 1st } \\
\text { year, Mayo } \\
\text { Medical } \\
\text { School }\end{array}$ & 44 & $\begin{array}{l}\text { To explore the } \\
\text { effectiveness of } \\
\text { using Google } \\
\text { online app } \\
\text { platform, a free } \\
\text { and widely- } \\
\text { accessible online } \\
\text { app platform to } \\
\text { manage and } \\
\text { teach a first-year } \\
\text { pathology } \\
\text { course }\end{array}$ & $\begin{array}{l}\text { The online app } \\
\text { platform allowed } \\
\text { faculty to build } \\
\text { an efficient and } \\
\text { effective } \\
\text { classroom } \\
\text { teaching and } \\
\text { management } \\
\text { system. The } \\
\text { ease of } \\
\text { accessibility and } \\
\text { opportunity for } \\
\text { collaboration } \\
\text { allowed for } \\
\text { collaborative } \\
\text { learning, grading, } \\
\text { and teaching. }\end{array}$ & Effectiveness \\
\hline $\begin{array}{l}\text { Diug, B., } \\
\text { et al. }\end{array}$ & 2016 & Australia & $\begin{array}{l}\text { Survey, Exam } \\
\text { grades }\end{array}$ & $\begin{array}{l}\text { biomedical } \\
\text { science, 1st } \\
\text { year, Monash } \\
\text { University }\end{array}$ & 236 & $\begin{array}{l}\text { To evaluate } \\
\text { whether social } \\
\text { media, in } \\
\text { particular } \\
\text { Twitter, can be } \\
\text { successfully } \\
\text { used as a } \\
\text { pedagogical tool } \\
\text { in an } \\
\text { assessment to } \\
\text { increase student } \\
\text { engagement } \\
\text { with staff, peers } \\
\text { and course } \\
\text { content }\end{array}$ & $\begin{array}{l}\text { Use of social } \\
\text { media as an } \\
\text { additional, or } \\
\text { alternate, } \\
\text { teaching } \\
\text { intervention is } \\
\text { positively } \\
\text { supported by } \\
\text { students. } \\
\text { Specific use of } \\
\text { micro-blogs } \\
\text { such as Twitter } \\
\text { can promote } \\
\text { greater } \\
\text { student-staff } \\
\text { engagement by } \\
\text { developing an } \\
\text { ongoing } \\
\text { academic } \\
\text { conversation. }\end{array}$ & Effectiveness \\
\hline $\begin{array}{l}\text { Avila, J., } \\
\text { et al. }\end{array}$ & 2016 & Switzerland & $\begin{array}{l}\text { Survey, Focus } \\
\text { Group, Data } \\
\text { analytics }\end{array}$ & $\begin{array}{l}\text { cardiovascular } \\
\text { system, } \\
\text { digestive } \\
\text { system, 2nd } \\
\text { year, University } \\
\text { of Zurich }\end{array}$ & 25 & $\begin{array}{l}\text { To design and } \\
\text { evaluate an } \\
\text { ePortfolio } \\
\text { system with } \\
\text { mobile } \\
\text { capabilities } \\
\text { using } \\
\text { WordPress, a } \\
\text { commercially } \\
\text { free and open } \\
\text { source software } \\
\text { solution } \\
\text { assessing } \\
\text { technical } \\
\text { implementation } \\
\text { and usability by } \\
\text { online } \\
\text { questionnaires } \\
\text { and focus } \\
\text { groups }\end{array}$ & $\begin{array}{l}\text { The } \\
\text { implementation } \\
\text { of WordPress } \\
\text { should be } \\
\text { accompanied by } \\
\text { introductory } \\
\text { courses in the } \\
\text { use of the } \\
\text { software and its } \\
\text { apps in order to } \\
\text { facilitate its } \\
\text { usability. }\end{array}$ & Ease of use \\
\hline
\end{tabular}




\begin{tabular}{|c|c|c|c|c|c|c|c|c|}
\hline Author & Year & Country & $\begin{array}{l}\text { Methods/ } \\
\text { Analysis }\end{array}$ & $\begin{array}{l}\text { Population } \\
\text { (course, year, } \\
\text { university) }\end{array}$ & Sample Size & $\begin{array}{l}\text { Aim/Purpose of } \\
\text { study }\end{array}$ & Conclusion & Focus \\
\hline $\begin{array}{l}\text { Mi, M. } \\
\text { and D. } \\
\text { Gould }\end{array}$ & 2014 & US & $\begin{array}{l}\text { Survey, Focus } \\
\text { Group }\end{array}$ & $\begin{array}{l}\text { neuroscience, } \\
\text { 1st year, } \\
\text { Oakland } \\
\text { University }\end{array}$ & 75 & $\begin{array}{l}\text { To provide a } \\
\text { formative } \\
\text { evaluation of the } \\
\text { wiki group } \\
\text { project and to } \\
\text { examine how } \\
\text { wiki technology } \\
\text { was utilized to } \\
\text { enhance active } \\
\text { and } \\
\text { collaborative } \\
\text { learning of first- } \\
\text { year medical } \\
\text { students in the } \\
\text { course and to } \\
\text { reinforce } \\
\text { information } \\
\text { literacy skills. }\end{array}$ & $\begin{array}{l}\text { Given its ease of } \\
\text { use and } \\
\text { facilitation of } \\
\text { collaborative } \\
\text { learning and } \\
\text { authorship, } \\
\text { Google Sites or } \\
\text { other wiki } \\
\text { products may be } \\
\text { leveraged as a } \\
\text { potentially } \\
\text { enriching } \\
\text { learning and } \\
\text { teaching tool to } \\
\text { promote active } \\
\text { learning in } \\
\text { medical } \\
\text { education or any } \\
\text { educational } \\
\text { setting. }\end{array}$ & $\begin{array}{l}\text { Learning } \\
\text { design }\end{array}$ \\
\hline $\begin{array}{l}\text { Hall, P., et } \\
\text { al. }\end{array}$ & 2012 & Canada & $\begin{array}{l}\text { Case study, } \\
\text { reflection }\end{array}$ & $\begin{array}{l}\text { medical } \\
\text { students, The } \\
\text { University of } \\
\text { Ottawa }\end{array}$ & 600 & $\begin{array}{l}\text { To describe the } \\
\text { evolving } \\
\text { ePortfolio } \\
\text { program, reflect } \\
\text { on the } \\
\text { challenges have } \\
\text { faced in its } \\
\text { development } \\
\text { and } \\
\text { implementation, } \\
\text { and share the } \\
\text { lessons have } \\
\text { learned on the } \\
\text { way to a } \\
\text { successful and } \\
\text { sustainable } \\
\text { program }\end{array}$ & $\begin{array}{l}\text { A successful and } \\
\text { sustainable } \\
\text { program should } \\
\text { be user-friendly, } \\
\text { ensure that } \\
\text { faculty and } \\
\text { students } \\
\text { understand the } \\
\text { value of the } \\
\text { ePortfolio } \\
\text { program, can } \\
\text { soliciting student } \\
\text { input to improve } \\
\text { the program and } \\
\text { increase student } \\
\text { buy-in; and } \\
\text { providing faculty } \\
\text { development } \\
\text { opportunities } \\
\text { and recognition. }\end{array}$ & $\begin{array}{l}\text { Learning } \\
\text { design }\end{array}$ \\
\hline $\begin{array}{l}\text { Bahner, } \\
\text { D. P., et } \\
\text { al. }\end{array}$ & 2012 & US & Survey & $\begin{array}{l}\text { followers on } \\
\text { Twitter and } \\
\text { Facebook }\end{array}$ & 27 & $\begin{array}{l}\text { To demonstrate } \\
\text { a supplement to } \\
\text { a curriculum } \\
\text { using "push } \\
\text { technology" via } \\
\text { Twitter and } \\
\text { Facebook to } \\
\text { deliver } \\
\text { educational } \\
\text { content to } \\
\text { mobile devices }\end{array}$ & $\begin{array}{l}\text { Due to ease of } \\
\text { use and } \\
\text { widespread } \\
\text { applicability, } \\
\text { Twitter and } \\
\text { Facebook are } \\
\text { excellent } \\
\text { applications of } \\
\text { "push } \\
\text { technology" as a } \\
\text { means to deliver } \\
\text { educational } \\
\text { content. This } \\
\text { pilot project } \\
\text { demonstrates } \\
\text { the potential of } \\
\text { social media to } \\
\text { both supplement } \\
\text { and enhance } \\
\text { traditional } \\
\text { educational } \\
\text { methods. }\end{array}$ & $\begin{array}{l}\text { Learning } \\
\text { design, } \\
\text { Effectiveness }\end{array}$ \\
\hline
\end{tabular}




\begin{tabular}{|c|c|c|c|c|c|c|c|c|}
\hline Author & Year & Country & $\begin{array}{l}\text { Methods/ } \\
\text { Analysis }\end{array}$ & $\begin{array}{l}\text { Population } \\
\text { (course, year, } \\
\text { university) }\end{array}$ & Sample Size & $\begin{array}{l}\text { Aim/Purpose of } \\
\text { study }\end{array}$ & Conclusion & Focus \\
\hline $\begin{array}{l}\text { Potts, H. } \\
\text { W. }\end{array}$ & 2011 & UK & $\begin{array}{l}\text { Interview, } \\
\text { Focus Group, } \\
\text { Ethnographic } \\
\text { data }\end{array}$ & $\begin{array}{l}\text { peer-assisted } \\
\text { learning, 5th } \\
\text { year \& } \\
\text { postgrad, } \\
\text { University } \\
\text { College } \\
\text { London, }\end{array}$ & 53 & $\begin{array}{l}\text { To understand } \\
\text { students' } \\
\text { affective and } \\
\text { other } \\
\text { experiences of } \\
\text { generating } \\
\text { content to be } \\
\text { shared online } \\
\text { with peers (e.g. } \\
\text { in online } \\
\text { discussions) by } \\
\text { online interviews, } \\
\text { focus groups, } \\
\text { research log and } \\
\text { transcripts }\end{array}$ & $\begin{array}{l}\text { Successfully } \\
\text { introducing user- } \\
\text { generated } \\
\text { content into } \\
\text { medical } \\
\text { education } \\
\text { requires } \\
\text { attention to } \\
\text { practical details } \\
\text { and an } \\
\text { awareness of the } \\
\text { student anxiety } \\
\text { that can arise. }\end{array}$ & $\begin{array}{l}\text { Enablers and } \\
\text { barriers }\end{array}$ \\
\hline $\begin{array}{l}\text { Varga- } \\
\text { Atkins, T., } \\
\text { et al. }\end{array}$ & 2010 & UK & $\begin{array}{l}\text { Data } \\
\text { analytics, } \\
\text { survey, focus } \\
\text { group, } \\
\text { interviews }\end{array}$ & $\begin{array}{l}\text { problem-based } \\
\text { learning (PBL), } \\
\text { 1st year, The } \\
\text { University of } \\
\text { Liverpool }\end{array}$ & 32 & $\begin{array}{l}\text { To explore the } \\
\text { effectiveness of } \\
\text { developing } \\
\text { professionalism } \\
\text { through the use } \\
\text { of wikis, using } \\
\text { survey, focus } \\
\text { groups, } \\
\text { facilitator } \\
\text { interviews and } \\
\text { wikis usage } \\
\text { statistics }\end{array}$ & $\begin{array}{l}\text { Wikis acted as a } \\
\text { shared } \\
\text { knowledge base } \\
\text { for hard-to-find } \\
\text { resources on } \\
\text { professionalism. } \\
\text { Second, it was } \\
\text { precisely when } \\
\text { students } \\
\text { reflected on the } \\
\text { difference } \\
\text { between } \\
\text { interacting in } \\
\text { wikis and their } \\
\text { online social } \\
\text { spaces, or when } \\
\text { they considered } \\
\text { whether or not to } \\
\text { post a resource } \\
\text { that their sense } \\
\text { of } \\
\text { professionalism } \\
\text { emerged. }\end{array}$ & Effectiveness \\
\hline $\begin{array}{l}\text { Chretien, } \\
\text { K., et al. }\end{array}$ & 2008 & US & $\begin{array}{l}\text { Content } \\
\text { analysis, } \\
\text { Survey }\end{array}$ & $\begin{array}{l}\text { basic medicine } \\
\text { clerkship } \\
\text { rotation, } \\
\text { George } \\
\text { Washington } \\
\text { University }\end{array}$ & 91 & $\begin{array}{l}\text { To explore the } \\
\text { use of blogs in } \\
\text { reflective writing } \\
\text { and peer } \\
\text { feedback on } \\
\text { professional } \\
\text { development } \\
\text { using } \\
\text { collaborative, } \\
\text { web-based } \\
\text { technology by } \\
\text { student } \\
\text { evaluation and } \\
\text { analysis of posts }\end{array}$ & $\begin{array}{l}\text { Blogs can } \\
\text { promote } \\
\text { reflection, } \\
\text { uncover } \\
\text { elements of the } \\
\text { hidden } \\
\text { curriculum, and } \\
\text { provide } \\
\text { opportunities to } \\
\text { promote } \\
\text { professional } \\
\text { development. }\end{array}$ & Effectiveness \\
\hline $\begin{array}{l}\text { Baker, P. } \\
\text { G., et al. }\end{array}$ & 2005 & Australia & $\begin{array}{l}\text { Content } \\
\text { analysis, } \\
\text { Survey }\end{array}$ & $\begin{array}{l}\text { rural medicine } \\
\text { rotation, 3rd } \\
\text { year, The } \\
\text { University of } \\
\text { Queensland }\end{array}$ & 83 & $\begin{array}{l}\text { To evaluate the } \\
\text { ease of use and } \\
\text { access and the } \\
\text { educational } \\
\text { value of a web- } \\
\text { based Clinical } \\
\text { Discussion } \\
\text { Board, and } \\
\text { interest in } \\
\text { pursuing a rural } \\
\text { medicine career } \\
\text { by survey and } \\
\text { transcripts on } \\
\text { CDB }\end{array}$ & $\begin{array}{l}\text { A web-based } \\
\text { Clinical } \\
\text { Discussion } \\
\text { Board offers a } \\
\text { unique way to } \\
\text { understand the } \\
\text { concerns and } \\
\text { interests of third } \\
\text { year medical } \\
\text { students. It } \\
\text { highlights the } \\
\text { issues they need } \\
\text { to discuss with } \\
\text { their peers, and } \\
\text { offers the } \\
\text { potential to } \\
\text { guide future } \\
\text { curriculum } \\
\text { changes in } \\
\text { response to } \\
\text { identified needs. }\end{array}$ & $\begin{array}{l}\text { Educational } \\
\text { value, Ease } \\
\text { of use }\end{array}$ \\
\hline
\end{tabular}




\begin{tabular}{|c|c|c|c|c|c|c|c|c|}
\hline Author & Year & Country & $\begin{array}{l}\text { Methods/ } \\
\text { Analysis }\end{array}$ & $\begin{array}{l}\text { Population } \\
\text { (course, year, } \\
\text { university) }\end{array}$ & Sample Size & $\begin{array}{l}\text { Aim/Purpose of } \\
\text { study }\end{array}$ & Conclusion & Focus \\
\hline $\begin{array}{l}\text { Taradi, S. } \\
\text { K. and M. } \\
\text { Taradi }\end{array}$ & 2004 & Croatia & $\begin{array}{l}\text { Data } \\
\text { analytics, } \\
\text { Questionnaire, } \\
\text { Exam grades }\end{array}$ & $\begin{array}{l}\text { physiology, } \\
\text { 2nd year, } \\
\text { University of } \\
\text { Zagreb } \\
\text { Medical } \\
\text { School }\end{array}$ & $55+195+220$ & $\begin{array}{l}\text { To describe the } \\
\text { use of a free, } \\
\text { asynchronous } \\
\text { online forum to } \\
\text { expand student- } \\
\text { teacher } \\
\text { discussions } \\
\text { beyond the } \\
\text { time/place } \\
\text { constraints of } \\
\text { the physical } \\
\text { physiology } \\
\text { classroom by } \\
\text { assessing the } \\
\text { administration } \\
\text { data, } \\
\text { questionnaire } \\
\text { and final exam }\end{array}$ & $\begin{array}{l}\text { Faculty vary } \\
\text { considerably in } \\
\text { both their } \\
\text { abilities and their } \\
\text { attitudes toward } \\
\text { the new } \\
\text { technologies, } \\
\text { and } \\
\text { institutionally } \\
\text { based attempts } \\
\text { to engage the } \\
\text { faculty must } \\
\text { take these } \\
\text { variations into } \\
\text { account to be } \\
\text { successful. }\end{array}$ & Effectiveness \\
\hline $\begin{array}{l}\text { Bernardo, } \\
\text { V., et al. }\end{array}$ & 2004 & Brazil & $\begin{array}{l}\text { Data } \\
\text { analytics, } \\
\text { Questionnaire, } \\
\text { Quiz }\end{array}$ & $\begin{array}{l}\text { experimental } \\
\text { surgery, 3rd } \\
\text { year, Federal } \\
\text { University of } \\
\text { São Paulo }\end{array}$ & 56 & $\begin{array}{l}\text { To describe and } \\
\text { discuss the } \\
\text { process and the } \\
\text { lessons learned } \\
\text { involved in } \\
\text { developing and } \\
\text { implementing a } \\
\text { web-based } \\
\text { course for UG } \\
\text { medical } \\
\text { students, and to } \\
\text { analyze the } \\
\text { students' } \\
\text { attitude and } \\
\text { receptivity } \\
\text { towards this } \\
\text { educational } \\
\text { environment by } \\
\text { accessing } \\
\text { knowledge gain, } \\
\text { web usage data, } \\
\text { and course } \\
\text { evaluation }\end{array}$ & $\begin{array}{l}\text { This study } \\
\text { indicated that a } \\
\text { web-based } \\
\text { course for } \\
\text { undergraduate } \\
\text { students may be } \\
\text { successfully } \\
\text { developed and } \\
\text { implemented in } \\
\text { medical settings } \\
\text { and the students } \\
\text { seem to be quite } \\
\text { supportive. We } \\
\text { encourage } \\
\text { undergraduate } \\
\text { medical learning } \\
\text { strategies } \\
\text { involving the } \\
\text { Web. }\end{array}$ & $\begin{array}{l}\text { Learning } \\
\text { design, } \\
\text { Effectiveness }\end{array}$ \\
\hline $\begin{array}{l}\text { Wiecha, } \\
\text { J. M., et } \\
\text { al. }\end{array}$ & 2003 & US & $\begin{array}{l}\text { Interview, } \\
\text { Focus Group, } \\
\text { Questionnaire }\end{array}$ & $\begin{array}{l}\text { medical } \\
\text { interviewing, } \\
\text { 1st \& 2nd year, } \\
\text { Boston } \\
\text { University and } \\
\text { University of } \\
\text { Massachusetts }\end{array}$ & 10 & $\begin{array}{l}\text { To evaluate a 4- } \\
\text { week online } \\
\text { elective course } \\
\text { for medical } \\
\text { students to } \\
\text { teach the } \\
\text { cognitive basis } \\
\text { for interviewing } \\
\text { skills, with a } \\
\text { moderated, } \\
\text { asynchronous } \\
\text { discussion board }\end{array}$ & $\begin{array}{l}\text { Online education } \\
\text { has significant } \\
\text { potential to } \\
\text { augment } \\
\text { curriculum on } \\
\text { the medical } \\
\text { interview, } \\
\text { particularly } \\
\text { among students } \\
\text { trained in } \\
\text { community } \\
\text { settings } \\
\text { geographically } \\
\text { distant from } \\
\text { their academic } \\
\text { medical center. }\end{array}$ & Effectiveness \\
\hline
\end{tabular}




\begin{tabular}{|c|c|c|c|c|c|c|c|c|}
\hline Author & Year & Country & $\begin{array}{l}\text { Methods/ } \\
\text { Analysis }\end{array}$ & $\begin{array}{l}\text { Population } \\
\text { (course, } \\
\text { year, } \\
\text { university) }\end{array}$ & $\begin{array}{l}\text { Sample } \\
\text { Size }\end{array}$ & $\begin{array}{l}\text { Aim/Purpose of } \\
\text { study }\end{array}$ & Conclusion & Focus \\
\hline \multicolumn{9}{|l|}{ Literature reviews } \\
\hline Reeves, S., et al. & 2017 & UK/Ireland & $\begin{array}{l}\text { Scoping } \\
\text { review }\end{array}$ & $\begin{array}{l}\text { Medline, } \\
\text { CINAHL, } \\
\text { BEl, } \\
\text { PubMed, } \\
\text { Scopus, } \\
\text { Web of } \\
\text { Science }\end{array}$ & $23 / 1568$ & $\begin{array}{l}\text { To review what } \\
\text { is the nature of } \\
\text { evidence on } \\
\text { online } \\
\text { postgraduate } \\
\text { education for } \\
\text { primary } \\
\text { healthcare inter- } \\
\text { professional } \\
\text { teams? } \\
\text { What learning } \\
\text { approaches and } \\
\text { study methods } \\
\text { are used in this } \\
\text { context? What is } \\
\text { the range of } \\
\text { reported } \\
\text { outcomes for } \\
\text { primary } \\
\text { healthcare } \\
\text { learners, their } \\
\text { organizations } \\
\text { and the care } \\
\text { they deliver to } \\
\text { patients/clients? }\end{array}$ & $\begin{array}{l}\text { E-learning can } \\
\text { enhance an } \\
\text { education } \\
\text { experience, } \\
\text { support } \\
\text { development, } \\
\text { ease time } \\
\text { constraints, } \\
\text { overcome } \\
\text { geographic } \\
\text { limitations and } \\
\text { can offer greater } \\
\text { flexibility. } \\
\text { However, it can } \\
\text { also contribute } \\
\text { to the isolation } \\
\text { of learners and } \\
\text { its benefits can } \\
\text { be negated by } \\
\text { technical } \\
\text { problems. }\end{array}$ & $\begin{array}{l}\text { Learning } \\
\text { design }\end{array}$ \\
\hline Guraya, S. & 2016 & Saudi Arabia & $\begin{array}{l}\text { Systematic } \\
\text { review }\end{array}$ & $\begin{array}{l}\text { ERIC, } \\
\text { CINAHL, } \\
\text { Cochrane } \\
\text { library, and } \\
\text { EMBASE }\end{array}$ & $10 / 1188$ & $\begin{array}{l}\text { To determine the } \\
\text { medical } \\
\text { students' extent } \\
\text { of usage of } \\
\text { SNSs for } \\
\text { educational } \\
\text { purposes }\end{array}$ & $\begin{array}{l}\text { Understanding } \\
\text { and knowledge } \\
\text { of the } \\
\text { significant use } \\
\text { of SNSs by the } \\
\text { medical } \\
\text { students } \\
\text { demand } \\
\text { inclusion of } \\
\text { such domains in } \\
\text { medical } \\
\text { curricula. This } \\
\text { will train } \\
\text { tomorrow's } \\
\text { doctors in } \\
\text { fostering their } \\
\text { skills of digital } \\
\text { technology for } \\
\text { educational } \\
\text { purposes. }\end{array}$ & $\begin{array}{l}\text { Prevalence of } \\
\text { usage }\end{array}$ \\
\hline $\begin{array}{l}\text { Batt-Rawden, S., } \\
\text { et al. }\end{array}$ & 2014 & US/UK/Australia & $\begin{array}{l}\text { Systematic } \\
\text { review }\end{array}$ & $\begin{array}{l}\text { MEDLINE, } \\
\text { CINAHL, } \\
\text { ERIC, } \\
\text { EMBASE, } \\
\text { PsycINFO, } \\
\text { Proquest, } \\
\text { Cochrane } \\
\text { Library, } \\
\text { Web of } \\
\text { Science } \\
\text { and } \\
\text { Scopus }\end{array}$ & $14 / 928$ & $\begin{array}{l}\text { To identify } \\
\text { examples of } \\
\text { how social } \\
\text { media may be } \\
\text { used to help } \\
\text { promote the } \\
\text { achievement of } \\
\text { clinical } \\
\text { excellence in } \\
\text { medical learners }\end{array}$ & $\begin{array}{l}\text { SM may help } \\
\text { facilitate the } \\
\text { achievement of } \\
\text { clinical } \\
\text { excellence. }\end{array}$ & $\begin{array}{l}\text { Learning } \\
\text { design }\end{array}$ \\
\hline
\end{tabular}




\begin{tabular}{|c|c|c|c|c|c|c|c|c|}
\hline Author & Year & Country & $\begin{array}{l}\text { Methods/ } \\
\text { Analysis }\end{array}$ & $\begin{array}{l}\text { Population } \\
\text { (course, } \\
\text { year, } \\
\text { university) }\end{array}$ & $\begin{array}{l}\text { Sample } \\
\text { Size }\end{array}$ & $\begin{array}{l}\text { Aim/Purpose of } \\
\text { study }\end{array}$ & Conclusion & Focus \\
\hline $\begin{array}{l}\text { Smith, T. and R. } \\
\text { Lambert }\end{array}$ & 2014 & UK & $\begin{array}{l}\text { Systematic } \\
\text { review }\end{array}$ & $\begin{array}{l}\text { AMED, } \\
\text { BNI, } \\
\text { CINAHL, } \\
\text { EMBASE, } \\
\text { MEDLINE, } \\
\text { PsycINFO, } \\
\text { PubMed, } \\
\text { the } \\
\text { Cochrane } \\
\text { Library, } \\
\text { Australian } \\
\text { Education } \\
\text { Index, and } \\
\text { British } \\
\text { Education } \\
\text { Index }\end{array}$ & $16 / 876$ & $\begin{array}{l}\text { To evaluate the } \\
\text { use, attitudes } \\
\text { and perceptions } \\
\text { of both teachers } \\
\text { and students } \\
\text { towards social } \\
\text { media platforms } \\
\text { (Facebook and } \\
\text { twitter) in } \\
\text { healthcare } \\
\text { higher education } \\
\text { practice }\end{array}$ & $\begin{array}{l}\text { Facebook and } \\
\text { Twitter are } \\
\text { perceived as } \\
\text { potentially } \\
\text { useful adjuncts } \\
\text { in healthcare } \\
\text { higher } \\
\text { education } \\
\text { practice, which } \\
\text { enhance } \\
\text { communication } \\
\text { and increase } \\
\text { accessibility of } \\
\text { students to real- } \\
\text { world practices } \\
\text { and expertise. } \\
\text { Whilst students } \\
\text { perceive this to } \\
\text { be of value, } \\
\text { some have } \\
\text { reservations } \\
\text { regarding the } \\
\text { possibility of } \\
\text { faculty } \\
\text { members spying } \\
\text { or infringing on } \\
\text { their "virtual" } \\
\text { social } \\
\text { community. } \\
\text { Faculty } \\
\text { members } \\
\text { expressed some } \\
\text { reservations } \\
\text { regarding the } \\
\text { perceived } \\
\text { boundary } \\
\text { between } \\
\text { student-staff } \\
\text { relationships. }\end{array}$ & $\begin{array}{l}\text { Educational } \\
\text { value, } \\
\text { Prevalence of } \\
\text { usage }\end{array}$ \\
\hline $\begin{array}{l}\text { Cheston, C. C., et } \\
\text { al. }\end{array}$ & 2013 & US & $\begin{array}{l}\text { Systematic } \\
\text { review }\end{array}$ & $\begin{array}{l}\text { MEDLINE, } \\
\text { Scopus, } \\
\text { Embase, } \\
\text { Cochrane } \\
\text { Library, } \\
\text { CINAHL, } \\
\text { Web of } \\
\text { Science, } \\
\text { PsycINFO, } \\
\text { ProQuest, } \\
\text { ERIC }\end{array}$ & $14 / 928$ & $\begin{array}{l}\text { To review how } \\
\text { have } \\
\text { interventions } \\
\text { using social } \\
\text { media tools } \\
\text { affected } \\
\text { outcomes of } \\
\text { satisfaction, } \\
\text { knowledge, } \\
\text { attitudes, and } \\
\text { skills for } \\
\text { physicians and } \\
\text { physicians-in } \\
\text { training? and } \\
\text { what challenges } \\
\text { and } \\
\text { opportunities } \\
\text { specific to social } \\
\text { media have } \\
\text { educators } \\
\text { encountered in } \\
\text { implementing } \\
\text { these } \\
\text { interventions? }\end{array}$ & $\begin{array}{l}\text { Social media } \\
\text { use in medical } \\
\text { education is an } \\
\text { emerging field } \\
\text { of scholarship } \\
\text { that merits } \\
\text { further } \\
\text { investigation. } \\
\text { Educators face } \\
\text { challenges in } \\
\text { adapting new } \\
\text { technologies, } \\
\text { but they also } \\
\text { have } \\
\text { opportunities for } \\
\text { innovation. }\end{array}$ & $\begin{array}{l}\text { Effectiveness, } \\
\text { Enablers and } \\
\text { barriers }\end{array}$ \\
\hline
\end{tabular}




\begin{tabular}{|c|c|c|c|c|c|c|c|c|}
\hline Author & Year & Country & $\begin{array}{l}\text { Methods/ } \\
\text { Analysis }\end{array}$ & $\begin{array}{l}\text { Population } \\
\text { (course, } \\
\text { year, } \\
\text { university) }\end{array}$ & $\begin{array}{l}\text { Sample } \\
\text { Size }\end{array}$ & $\begin{array}{l}\text { Aim/Purpose of } \\
\text { study }\end{array}$ & Conclusion & Focus \\
\hline $\begin{array}{l}\text { Hollinderbäumer, } \\
\text { A., et al. }\end{array}$ & 2013 & Germany & $\begin{array}{l}\text { Systematic } \\
\text { review }\end{array}$ & PubMed & $20 / 1245$ & $\begin{array}{l}\text { To summarize } \\
\text { the manner in } \\
\text { which the } \\
\text { integration of } \\
\text { Social Media } \\
\text { and Web } 2.0 \\
\text { into education }\end{array}$ & $\begin{array}{l}\text { The integration } \\
\text { of Web } 2.0 \text { and } \\
\text { Social Media is } \\
\text { the modern } \\
\text { form of self- } \\
\text { determined } \\
\text { learning. It } \\
\text { stimulates } \\
\text { reflection and } \\
\text { actively } \\
\text { integrates the } \\
\text { students in the } \\
\text { construction of } \\
\text { their knowledge. } \\
\text { With these new } \\
\text { tools, the } \\
\text { students acquire } \\
\text { skills which they } \\
\text { need in both } \\
\text { their social and } \\
\text { professional } \\
\text { lives. }\end{array}$ & $\begin{array}{l}\text { Learning } \\
\text { design, } \\
\text { Effectiveness }\end{array}$ \\
\hline $\begin{array}{l}\text { Cartledge, P., et } \\
\text { al. }\end{array}$ & 2013 & UK/Rwanda & $\begin{array}{l}\text { Systematic } \\
\text { review }\end{array}$ & $\begin{array}{l}\text { Medline, } \\
\text { Embase, } \\
\text { PsycINFO, } \\
\text { ERIC, } \\
\text { CINAHL, } \\
\text { Cochrane } \\
\text { library, BIE, } \\
\text { RDRBWEB }\end{array}$ & 9/1047 & $\begin{array}{l}\text { To ascertain if } \\
\text { social } \\
\text { networking sites } \\
\text { have been used } \\
\text { successfully in } \\
\text { medical } \\
\text { education to } \\
\text { deliver } \\
\text { educational } \\
\text { material, and } \\
\text { whether } \\
\text { healthcare } \\
\text { professionals, } \\
\text { and students, } \\
\text { are engaging } \\
\text { with social } \\
\text { networking sites } \\
\text { for educational } \\
\text { purposes }\end{array}$ & $\begin{array}{l}\text { Social- } \\
\text { networking sites } \\
\text { have been } \\
\text { employed } \\
\text { without } \\
\text { problems of } \\
\text { professionalism, } \\
\text { and received } \\
\text { positive } \\
\text { feedback from } \\
\text { learners. } \\
\text { However, there } \\
\text { is no solid } \\
\text { evidence base } \\
\text { within the } \\
\text { literature that } \\
\text { social- } \\
\text { networking is } \\
\text { equally or more } \\
\text { effective than } \\
\text { other media } \\
\text { available for } \\
\text { educational } \\
\text { purposes. }\end{array}$ & $\begin{array}{l}\text { Educational } \\
\text { value, } \\
\text { Effectiveness }\end{array}$ \\
\hline
\end{tabular}




\begin{tabular}{|c|c|c|c|c|c|}
\hline Author & Year & Country & Aim/Purpose of study & Conclusion & Focus \\
\hline \multicolumn{6}{|c|}{ Expert perspectives } \\
\hline $\begin{array}{l}\text { Hammerling, J. } \\
\text { A. M. S. H. M. S. } \\
\text { M. L. S. }\end{array}$ & 2012 & US & $\begin{array}{l}\text { To discuss the best practices of course } \\
\text { design and successful delivery of online } \\
\text { clinical laboratory science (CLS) courses, } \\
\text { and informs educators about the effective } \\
\text { use of currently available educational } \\
\text { technology tools used to increase student } \\
\text { engagement }\end{array}$ & $\begin{array}{l}\text { Technology has provided the tools for } \\
\text { distance learning, and online education } \\
\text { reaches more students than } \\
\text { conventional classroom education } \\
\text { can. }\end{array}$ & $\begin{array}{l}\text { Learning } \\
\text { design, } \\
\text { Enablers and } \\
\text { barriers }\end{array}$ \\
\hline $\begin{array}{l}\text { Popoiu, M. C., et } \\
\text { al. }\end{array}$ & 2012 & Romania & $\begin{array}{l}\text { To promote scholarly inquiry about the } \\
\text { development and adoption of best practice } \\
\text { in teaching and learning in medical } \\
\text { education with social media }\end{array}$ & $\begin{array}{l}\text { There is an increased need for training } \\
\text { in how to use these technologies to } \\
\text { enhance teaching and learning to } \\
\text { support undergraduate and } \\
\text { postgraduate medical education. }\end{array}$ & $\begin{array}{l}\text { Educational } \\
\text { value, } \\
\text { Enablers and } \\
\text { barriers }\end{array}$ \\
\hline Wells, K. M. & 2011 & US & $\begin{array}{l}\text { To share the author's own experience and } \\
\text { observation in using social media for } \\
\text { communication, up-to-date knowledge, life- } \\
\text { long learning related to medical education }\end{array}$ & $\begin{array}{l}\text { Social media will shape general } \\
\text { surgery and surgical specialties } \\
\text { through shared information among the } \\
\text { global community of surgery }\end{array}$ & $\begin{array}{l}\text { Educational } \\
\text { value }\end{array}$ \\
\hline $\begin{array}{l}\text { McGee, J. B. } \\
\text { and M. Begg }\end{array}$ & 2008 & US/UK & $\begin{array}{l}\text { To review the unique features of Web } 2.0 \\
\text { technologies, addresses questions } \\
\text { regarding potential pitfalls and suggests } \\
\text { valuable applications in health science } \\
\text { education. }\end{array}$ & $\begin{array}{l}\text { Web } 2.0 \text { gives educators an } \\
\text { opportunity to use the Internet to craft } \\
\text { engaging, student-focused, self- } \\
\text { generating and sustainable learning } \\
\text { experiences. Web } 2.0 \text { has the potential } \\
\text { to fundamentally change the } \\
\text { relationship between teacher and } \\
\text { student. }\end{array}$ & $\begin{array}{l}\text { Enablers and } \\
\text { barriers }\end{array}$ \\
\hline $\begin{array}{l}\text { McLean, R., et } \\
\text { al. }\end{array}$ & 2007 & UK & $\begin{array}{l}\text { To discuss the use of web } 2.0 \text { in medical } \\
\text { education in providing health information } \\
\text { "any time, any place" }\end{array}$ & $\begin{array}{l}\text { The medical community needs to be } \\
\text { aware of these Web } 2.0 \text { technologies } \\
\text { and their increasing role in providing } \\
\text { health information "any time, any } \\
\text { place". }\end{array}$ & $\begin{array}{l}\text { Educational } \\
\text { value }\end{array}$ \\
\hline $\begin{array}{l}\text { Sandars, J. and } \\
\text { C. } \\
\text { Haythornthwaite }\end{array}$ & 2007 & UK/USA & $\begin{array}{l}\text { To discuss how web } 2.0 \text { offers new } \\
\text { learning opportunities, importance of } \\
\text { developing connections between a wide } \\
\text { variety of learning resources }\end{array}$ & $\begin{array}{l}\text { New adaptive technology has the } \\
\text { potential to create personalized, yet } \\
\text { collective, learning. The future } \\
\text { implications for e-learning in medical } \\
\text { education is considered. }\end{array}$ & $\begin{array}{l}\text { Educational } \\
\text { value }\end{array}$ \\
\hline $\begin{array}{l}\text { Kamel Boulos, } \\
\text { M. N. and S. } \\
\text { Wheeler }\end{array}$ & 2007 & UK & $\begin{array}{l}\text { To discuss the use of web } 2.0 \text { in health and } \\
\text { health care education }\end{array}$ & $\begin{array}{l}\text { There is a need to raise awareness of } \\
\text { Web } 2.0 \text { tools and the possibilities they } \\
\text { offer, and an urgent need to conduct } \\
\text { quality research to inform better use of } \\
\text { Web } 2.0 \text { applications. }\end{array}$ & $\begin{array}{l}\text { Educational } \\
\text { value }\end{array}$ \\
\hline Boulos, M., et al. & 2006 & UK & $\begin{array}{l}\text { To explore some of the current uses of Web } \\
2.0 \text { tools in the education of } \\
\text { medical/nursing students, the continuing } \\
\text { professional development and education } \\
\text { of healthcare professionals, and patient } \\
\text { education }\end{array}$ & $\begin{array}{l}\text { If effectively deployed, wikis, blogs and } \\
\text { podcasts could offer a way to enhance } \\
\text { students', clinicians' and patients' } \\
\text { learning experiences, and deepen } \\
\text { levels of learners' engagement and } \\
\text { collaboration within digital learning } \\
\text { environments. }\end{array}$ & $\begin{array}{l}\text { Learning } \\
\text { design, } \\
\text { Effectiveness }\end{array}$ \\
\hline
\end{tabular}

Table 1

Summary of selected articles

Of the 36 included articles, 13 originated in the UK, 11 in the USA, the rest from Australia (three), Brazil (one), Canada (one), Croatia (one), Germany (one), Romania (one), Saudi Arabia (two), Singapore (one), and Switzerland (one). Five collaborative papers were led by the UK and the USA, supported by Australia, Ireland, and Rwanda.

A wide range of journals accepted papers on collaborative online learning in medical education led by Medical Teacher (9 papers), BMC Medical Education (3 papers), and Academic Medicine (2 papers). (Table 2) Medical educators are encouraged to publish their works and share their practical experience with other medical educators.

Table 2. Journal publications of selected articles 


\begin{tabular}{|c|c|c|}
\hline $\begin{array}{l}\text { Journal } \\
\text { Publication }\end{array}$ & Authors & \begin{tabular}{|l|}
$\begin{array}{l}\text { Number } \\
\text { of } \\
\text { articles }\end{array}$ \\
\end{tabular} \\
\hline $\begin{array}{l}\text { Academic } \\
\text { Medicine }\end{array}$ & Hall et al. (2012), Cheston et al. (2013) & 2 \\
\hline $\begin{array}{l}\text { Advances in } \\
\text { Physiology } \\
\text { Education }\end{array}$ & Taradi and Taradi (2004) & 1 \\
\hline $\begin{array}{l}\text { BMC Medical } \\
\text { Education }\end{array}$ & Boulos et al. (2006), Avila et al. (2016), Cole et al. (2017) & 3 \\
\hline BMJ Open & Reeves et al. (2017) & 1 \\
\hline Clinical Teacher & Batt-Rawden et al. (2014) & 1 \\
\hline $\begin{array}{l}\text { Education and } \\
\text { Information } \\
\text { Technologies }\end{array}$ & Dommett (2019) & 1 \\
\hline $\begin{array}{l}\text { Education for } \\
\text { Health }\end{array}$ & Diug et al. (2016) & 1 \\
\hline $\begin{array}{l}\text { GMS Z Med } \\
\text { Ausbild }\end{array}$ & Hollinderbäumer et al. (2013) & 1 \\
\hline Health Education & Smith and Lambert (2014) & 1 \\
\hline Health Info Libr & Kamel Boulos and Wheeler (2007) & 1 \\
\hline Int J Med Inform & Bernardo et al. (2004) & 1 \\
\hline $\begin{array}{l}\text { Journal of General } \\
\text { Internal Medicine }\end{array}$ & Chretien et al. (2008) & 1 \\
\hline $\begin{array}{l}\text { Journal of Medical } \\
\text { Internet Research }\end{array}$ & Wiecha et al. (2003) & 1 \\
\hline $\begin{array}{l}\text { Journal of the } \\
\text { Medical Library } \\
\text { Association }\end{array}$ & Lemley and Burnham (2009) & 1 \\
\hline Labmedicine & Hammerling (2012) & 1 \\
\hline Med Ref Serv & Mi and Gould (2014) & 1 \\
\hline Medical Education & Ho et al. (2018) & 1 \\
\hline $\begin{array}{l}\text { Medical Journal of } \\
\text { Australia }\end{array}$ & McLean et al. (2007) & 1 \\
\hline Medical Teacher & $\begin{array}{l}\text { Bahner et al. (2012), Cartledge et al. (2013), Gray et al. (2010), McGee and Begg (2008), } \\
\text { Peacock and Grande (2016), Potts (2011), Sandars and Haythornthwaite (2007), Sandars et al. } \\
\text { (2008), Varga-Atkins et al. (2010) }\end{array}$ & 9 \\
\hline $\begin{array}{l}\text { North American } \\
\text { Journal of Medical } \\
\text { Sciences }\end{array}$ & Guraya (2016) & 1 \\
\hline PLoS One & Saqr, et al. (2018) & 1 \\
\hline Postgrad Med J & Sandars and Schroter (2007) & 1 \\
\hline $\begin{array}{l}\text { Procedia - Social } \\
\text { and Behavioral } \\
\text { Sciences }\end{array}$ & Popoiu et al. (2012) & 1 \\
\hline $\begin{array}{l}\text { Rural Remote } \\
\text { Health }\end{array}$ & Baker et al. (2005) & 1 \\
\hline Surgery & Wells (2011) & 1 \\
\hline
\end{tabular}

\section{Description of Study Population}

Among the five studies of surveys on the usage of collaborative online learning, two studies were focused on members of medical associations, in which some members are undergraduate medical students, three studies are based on samples of students in one single university. The sample size ranged from 50 participants to 759 participants generally, with larger sample sizes for surveys than interventional studies. (Table 1)

There were 16 interventional studies on collaborative online learning. There was a wide range of study populations in the studies. Fourteen were based on an intervention at a single university, one was conducted in two different universities, and one targeted a more general population of followers on Twitter and Facebook including medical students. The sample size ranged from 10 participants to 300 participants. While single site studies are easier to conduct it is encouraging to know that a collaborative studies have been undertaken to provide more robust data on effectiveness and validity.

The interventions reported on a variety of applications in different courses. Eight interventions focused on a particular area of study, including a clinical clerkship, biomedical science, cardiovascular system and digestive system, experimental surgery, neuroscience, pathology, physiology, and rural medicine. Two focused on more generic clinical skills, including clinical reasoning, and medical interviewing. Three interventions focused on particular teaching and learning pedagogies, including case-based learning, peer-assistant learning, and problem-based learning. Among the interventional studies, nine studies involved students in the junior years (Year 1 and 2), and four studies involved students in the senior years (Year 3 or above).

\section{Methodological Approaches}


The outcome measure of collaborative online learning can be measured by 1) Self-reported satisfaction, 2) Self-reported knowledge gain, 3) Selfreported confidence gain, 4) Self-reported interests in pursuing certain career path, 5) Course evaluation, 6) Data analytics on platform usage (access, posting, duration), 7) Qualitative analysis of content (posts, comments, reactions), 8) Group interaction, student engagement, 9) completion rate of activities, and 10) Academic outcome.

\section{Key Insights from the Literature}

Several key educational issues emerged from this review: how collaborative online learning can influence learning (pedagogy), what modalities are useful in delivering collaborative online learning effectively (e-platforms and apps) and what are the enablers and barriers towards implementation. (Table 1)

\section{Pedagogical Perspectives}

\section{- Educational Value}

Many selected articles discussed the educational value of collaborative online learning and recognized its importance in medical education. Lemley \& Burnham pointed out the increasing trend of using collaborative online learning in medical education that encourages "anytime anyplace learning", personal engagement that suit preferred learning styles (3).

The novelty of using online learning generated interest in learning among students (18). Sandars \& Haythornthwaite highlighted the capacity of this learning module provide personalized learning that fit individual learning needs(16). Baker, Eley, \& Lasserre described how the online teaching tools they used helped them to understand students' concerns and interests in their learning, and hence provide useful guidance for curriculum development and career support.

The online mode of learning encouraged more interaction and sharing among students and teachers. It also helped build professional networks (18, $20)$, facilitated curriculum development $(15,18)$ and helped introduce future career paths $(18)$.

The dynamic virtual learning environment provided by collaborative learning applications encouraged student engagement in discussions and active learning. Cheston stated in his review that ten studies reported the use of social media in online learning stimulated interaction and promoted active learning (27).

- Learning Design

Nine articles discussed the learning design of collaborative online learning. Among these, one study suggested that the improved social interaction and sense of community belonging was a feature that made collaborative online learning effective (13). This can be undermined by a complicated and cumbersome design which underscores the importance of the selection of an appropriate application with a user friendly interface (15). Students are concerned about the extra workload imposed by collaborative online learning $(9,10)$ so ensuring that there is sufficient support is a crucial factor in successful online learning design (15). An example of support is pre-launch training and technical support targeting learners and educators with less confidence or ability in using online applications $(4,15,27,28)$.

- Learning effectiveness

Fifteen papers discussed the effectiveness of using collaborative online learning in medical education. Studies showed the sound effectiveness of collaborative online learning $(2,4)$, particularly when using social media (27), forum (13), discussion forum (14).

\section{- E-platforms and Applications}

There is a spectrum of e-platforms and applications, or apps, for collaborative online learning. These include blogs, document management systems, instant messaging, learning management systems, media sharing, online discussion, online games, social bookmarking, social networking and wikis. Social networking applications are most frequently discussed in and Facebook and Twitter are the most commonly used. Most of the used applications are commercially developed, with only 1 using a self-developed site for collaborative online learning. (Table 3)

Table 3. Variation of collaborative online learning applications used or discussed in the selected articles 


\begin{tabular}{|c|c|c|}
\hline Category & Examples & $\begin{array}{l}\text { Number of } \\
\text { papers }\end{array}$ \\
\hline Blogs & Blogger, Wordpress & 16 \\
\hline $\begin{array}{l}\text { Document management } \\
\text { system }\end{array}$ & $\begin{array}{l}\text { Google Suite (Google Doc, Google Drive, Google Form, Google Sheets, Google } \\
\text { Slides), SharePoint }\end{array}$ & 6 \\
\hline Instant messaging & Messenger, Skype & 5 \\
\hline $\begin{array}{l}\text { Learning management } \\
\text { system }\end{array}$ & $\begin{array}{l}\text { Nicenet's Internet Classroom Assistant (ICA; http://www.nicenet.org) Blackboard, } \\
\text { Moodle, Flubaroo, }\end{array}$ & 10 \\
\hline Media sharing & Podcast, Videocast (YouTube), Photo sharing (Flickr) & 18 \\
\hline Online discussion & Chatroom & 3 \\
\hline Online game & Dumb Ways to Die & 2 \\
\hline Social bookmarking & Pinterest, Scoop.it, Del.i.cious & 7 \\
\hline Social networking & Facebook, Twitter, LinkedIn, Myspace & 30 \\
\hline Wikis & Wikipedia, Google Sites, TeamsLX & 17 \\
\hline Other & Self-developed site & 1 \\
\hline
\end{tabular}

Seven studies focused on prevalence of using a particular application in collaborative online learning. Studies found that collaborative online learning applications are useful for knowledge acquisition $(29,30)$.

- Enablers and Barriers

This review found that 6 of the 36 included studies provided reports of the enablers and barriers for using collaborative online learning. Enablers include the accessibility of "any time, any place" learning in the online space provided powerful learning experiences for teachers and learners (18, $31,32)$. Collaborative online learning connects people with common interests $(29,31,33)$. Two articles described the ease of using collaborative online learning. The familiarity in using social media is not only easy but also enables learners to share information online freely (18, 29). A further study echoes that the ease of using is an important factor and advantage of successful online learning (34). Among these, several studies also pointed out the cost-effectiveness of using collaborative online learning $(2,4,18,35)$.

One of the key barriers is the technical challenges (29). The ever-evolving online technology provided a novel platform for learning. Though most students in the Web 2.0 generation understand how to use online learning (4) and most applications require little or minimal technical expertise (28, 36). Using collaborative online learning platforms demand some competence in handling technical techniques (14, 37). Appropriate technical support is still necessary (38). Failure of software and connection to online learning platform (18), un-familiarization of the application (39), the requirement of extra technical supports (2), and time and manpower to develop collaborative learning (14) are technical challenges of using collaborative online learning.

Other barriers of collaborative online learning including the concern of privacy issues (29), quality of information available online (28), difficulties with access to information online (28), learners' self-discipline (34). In contrast, some also pointed out that cost is a barrier in using such learning strategies $(2,27,37)$

\section{Discussion}

We sought to answer two questions with this scoping review:

- What is the extent and nature of the academic publications on collaborative online learning in medical education?

- What insights about collaborative online learning have we learned from the literature

\section{The Extent of Research Studies}

The 16 empirical studies of implementation showed that there is an increasing trend of using collaborative online learning in medical education but the relatively low prevalence of articles may suggest that medical educators are hesitant to adopt it until its effectiveness is well-proven or until the technological and pedagogical barriers are overcome. On the other hand, the steady number of papers published each year, shows that there is ongoing interest and implementation of this learning mode. In particular, there is potential for furthering its use in specialty-specific courses, medical skills training, and pedagogical development.

Page 17/21 
The majority of relevant studies were conducted in the UK and the USA, which may indicate that online learning started earlier, became more popular and gained more attention in those countries. The absence of empirical studies from Asia, Europe, and other regions is surprising, given the high smartphone penetration and high rate of technology adoption in Asian countries, for example. It may be that such innovations have just not been formally evaluated and published as this review was restricted to English language publication and did not cover the papers presented in medical education conferences and web-based publication. On the other hand it might also signify the potential of exploring how collaborative online learning in medical education is used in different cultural settings. The internet has no boundary, and online learning also has no geographical restriction which creates immense potential for collaborative work to explore how collaborative online learning in medical education can be pushed beyond current practices. Medical educators are encouraged to publish their work as a means of dissemination, networking and globalizing medical education.

\section{The Nature of Research Studies}

From this review, we found that the studies on the topic have been limited in scope with mostly small scale studies involving relatively small sample sizes. These may be pilot studies and perhaps some unsuccessful studies might not be reported. However, as the nature of online learning is geared towards large populations of learners, there is good potential for future interventions involving more learners resulting in studies with better validity for extrapolation and generalization of findings.

Our review showed that intervention studies are more popular in junior years than in senior years in medical school. This might be due to the junior years are more relevant and suitable for online learning, which can cater to larger classes. This indicated that the novel online learning pedagogy is more popular among the foundation years as it gives more space for teachers and learners to explore new technology that could enhance their learning experience. In the clerkship years, the nature of teaching would require more patient-based learning and require more face-to-face contact. However, with the recent COVID-19 outbreak, many institutions have been forced to find alternatives to face-to-face teaching and there may be many new collaborative online learning innovations born from this crisis in both clerkship and pre-clerkship settings.

A range of qualitative or quantitative research methods were used in the studies included in this review including one that used social network analysis (SNA) to understand the effectiveness of collaborative online learning. This approach provided a visual analysis of how learners collaborate online and helped to identify problem areas that require further attention and intervention. New research questions and corresponding research methods and approaches to analysis, such as SNA, adopted from other disciplines or domains, can help to understand the issues from other perspectives and further the scope of research.

As suggested by some reviewed articles, it would be interesting to expand the research in collaborative online learning by exploring how students interact with each other. This is an era of Web 2.0 and 3.0, researchers and medical educators must be upfront to the emerging development of technology and keep abreast of the demands and expectations of the learners. The exploration of new applications that fit the needs of online learners is crucial. (36)

\section{Strengths and weaknesses}

This scoping review included many empirical studies, which show the current usage of collaborative online learning in medical education and provide good insights into understanding how the intervention works. Those large scale surveys provided a macro-view on the extent of usage of collaborative online learning in medical education. The literature reviews on the topic provided an overview of the existing literature related to the topic. Many scoping reviews exclude opinion papers and commentaries, however, they are the ones that can give a more general understanding of the issue and provide an overview of how collaborative online learning is situated in the medical education and medical profession. Limitations of this scoping review include: absence of any quality assessment of the selected articles so there was no relative weighting or importance among the papers, restriction to English language papers, exclusion of conference papers and web-based publications, difficulty with synthesis of information due to the heterogeneity of sources.

\section{Conclusions}

Collaborative online learning has good educational value that can encourage active learning and enhance student engagement. The selected studies highlighted the importance of learning design, especially providing sufficient support for learners and teachers when introducing new technology to optimize the learning experience and outcomes. Social networking applications have a leading role. The exploration of using a commercially developed application and adopting its function for education would be a challenging but rewarding pedagogical breakthrough. Technological challenges remain a notable and constant barrier for collaborative online learning and will continue to require ongoing support for teachers and learners. With the relatively small body of work currently published on collaborative online learning in medical education, further work and innovation in this area may be forthcoming in response to the need for alternatives to traditional learning particularly in times of crisis.

\section{Abbreviations}

BEME Best Evidence Medical Education 
CME continuing medical education

MeSH Medical Subject Headings

SNA social network analysis

\section{Declarations}

\section{Ethics approval and consent to participate}

Not applicable

\section{Consent for publication}

Not applicable

\section{Availability of data and materials}

The datasets used and/or analysed during the current study are available from the corresponding author on reasonable request.

\section{Competing interests}

The authors declare that they have no competing interests.

\section{Funding}

This project was funded by the Hong Kong University Grants Committee (UGC) Funding Scheme for Teaching and Learning Related Proposals (2016-19 Triennium).

\section{Authors' contributions}

PL contributed to the design and implementation of the research, analysis of the results and was a major contributor in writing the manuscript. JT, $\mathrm{HT}, \mathrm{KC}$ performed the literature reviews of the articles. JC was involved in editing and overall supervision of the project. All authors read and approved the final manuscript.

\section{Acknowledgements}

Not applicable

\section{References}

1. Auyeung LH. Building a Collaborative Online Learning Community: A Case Study in Hong Kong. Journal of Educational Computing Research. 2004;31(2):119-36.

2. Reeves S, Fletcher S, McLoughlin C, Yim A, Patel KD. Interprofessional online learning for primary healthcare: findings from a scoping review. BMJ Open. 2017;7(8):e016872.

3. Lemley T, Burnham J. Web 2.0 tools in medical and nursing school curricula* *. Journal of the Medical Library Association. 2009;97(1):50-2.

4. Hammerling JAMSHMSMLS. Best Practices in Undergraduate Clinical Laboratory Science Online Education and Effective Use of Educational Technology Tools. Labmedicine. 2012;43(6):313-9.

5. Chretien K, Goldman E, Faselis C. The Reflective Writing Class Blog: Using Technology to Promote Reflection and Professional Development. Journal of General Internal Medicine. 2008;23(12):2066-70.

6. Peacock JG, Grande JP. An online app platform enhances collaborative medical student group learning and classroom management. Med Teach. 2016;38(2):174-80.

7. Bahner DP, Adkins E, Patel N, Donley C, Nagel R, Kman NE. How we use social media to supplement a novel curriculum in medical education. Medical Teacher. 2012;34(6):439-44.

8. Ho A, Hee N, Teo R. Facilitating co-learning of medical ethical issues using Facebook. Medical Education. 2018;52(5):560-1.

9. Mi M, Gould D. Wiki technology enhanced group project to promote active learning in a neuroscience course for first-year medical students: an exploratory study. Medical reference services quarterly. 2014;33(2):125-35.

10. Potts HW. Student experiences of creating and sharing material in online learning. Medical Teacher. 2011;33(11):e607-14.

11. Saqr M, Fors U, Tedre M, Nouri J. How social network analysis can be used to monitor online collaborative learning and guide an informed intervention. PLoS One. 2018;13(3). 
12. Diug B, Kendal E, llic D. Evaluating the use of twitter as a tool to increase engagement in medical education. Education for Health 2016;29(3):223-30.

13. Taradi SK, Taradi M. Expanding the traditional physiology class with asynchronous online discussions and collaborative projects. Advances in Physiology Education. 2004;28(1-4):73-8.

14. Bernardo V, Ramos MP, Plapler H, De Figueiredo LF, Nader HB, Ancao MS, et al. Web-based learning in undergraduate medical education: development and assessment of an online course on experimental surgery. International journal of medical informatics. 2004;73(9-10):731-42.

15. Hall P, Byszewski A, Sutherland S, Stodel EJ. Developing a sustainable electronic Portfolio (ePortfolio) program that fosters reflective practice and incorporates CanMEDS competencies into the undergraduate medical curriculum. Academic Medicine. 2012;87(6):744-51.

16. Sandars J, Haythornthwaite C. New horizons for e-learning in medical education: ecological and Web 2.0 perspectives. Medical Teacher. 2007;29(4):307-10.

17. Jeffrey L, Hegarty B, Kelly O, Penman M, Coburn D, McDonald J. Developing Digital Information Literacy in Higher Education: Obstacles and Supports. Journal of Information Technology Education. 2011;10:383-413.

18. Baker PG, Eley DS, Lasserre KE. Tradition and technology: teaching rural medicine using an internet discussion board. Rural and remote health. 2005;5(4):435.

19. Wiecha J, Barrie N. Collaborative online learning: a new approach to distance CME. Academic Medicine. 2002;77(9):928-9.

20. Batt-Rawden S, Flickinger T, Weiner J, Cheston C, Chisolm M. The role of social media in clinical excellence. The clinical teacher. 2014;11(4):264-9.

21. Guraya S. The usage of social networking sites by medical students for educational purposes: A meta-analysis and systematic review. North American Journal of Medical Sciences. 2016;8(7):268-78.

22. Cartledge P, Miller M, Phillips B. The use of social-networking sites in medical education. Medical Teacher. 2013;35(10):847-57.

23. Fontaine G, Lavallée A, Maheu-Cadotte M-A, Bouix-Picasso J, Bourbonnais A. Health science communication strategies used by researchers with the public in the digital and social media ecosystem: a systematic scoping review protocol. BMJ Open. 2018;8(1).

24. BEME. A BEME review: definition and description: Best Evidence Medical and Health Professional Education (BEME); [Available from: https://www.bemecollaboration.org/What+is+a+BEME+Review/.

25. Arksey H, O'Malley L. Scoping studies: towards a methodological framework. International Journal of Social Research Methodology. 2005;8(1):19-32.

26. Silverman BG. Computer supported collaborative learning. Computers Educ. 1995;25(3):81-91.

27. Cheston CC, Flickinger TE, Chisolm MS. Social media use in medical education: a systematic review. Academic Medicine. 2013;88(6):893-901.

28. Sandars J, Schroter S. Web 2.0 technologies for undergraduate and postgraduate medical education: an online survey. Postgraduate medical journal. 2007;83(986):759-62.

29. Smith T, Lambert R. A systematic review investigating the use of Twitter and Facebook in university-based healthcare education. Health Education. 2014;114(5):347-66.

30. Dommett EJ. Understanding Student Use of Twitter and Online Forums in Higher Education. Education and Information Technologies. 2019;24(1):325-43.

31. McLean R, Richards BH, Wardman Jl. The effect of Web 2.0 on the future of medical practice and education: Darwikinian evolution or folksonomic revolution? Medical Journal of Australia. 2007;187(3):174-7.

32. Boulos M, Maramba I, Wheeler S. Wikis, blogs and podcasts: a new generation of Web-based tools for virtual collaborative clinical practice and education. BMC Medical Education. 2006;6(1):41.

33. Kamel Boulos MN, Wheeler S. The emerging Web 2.0 social software: an enabling suite of sociable technologies in health and health care education. Health information and libraries journal. 2007;24(1):2-23.

34. Gray K, Annabell L, Kennedy G. Medical students' use of Facebook to support learning: Insights from four case studies. Medical Teacher. 2010;32(12):971-6.

35. Wells KM. Social media in medical school education. Surgery. 2011;150(1):2-4.

36. Sandars J, Homer M, Pell G, Croker T. Web 2.0 and social software: the medical student way of e-learning. Med Teach. 2008;30(3):308-12.

37. Avila J, Sostmann K, Breckwoldt J, Peters H. Evaluation of the free, open source software WordPress as electronic portfolio system in undergraduate medical education. BMC Medical Education. 2016;16:157.

38. McGee JB, Begg M. What medical educators need to know about "Web 2.0". Medical Teacher. 2008;30(2):164-9.

39. Varga-Atkins T, Dangerfield P, Brigden D. Developing professionalism through the use of wikis: A study with first-year undergraduate medical students. Med Teach. 2010;32(10):824-9.

\section{Figures}


Additional records identified through other sources $(\mathrm{n}=90)$


Records after duplicates removed $(\mathrm{n}=51)$
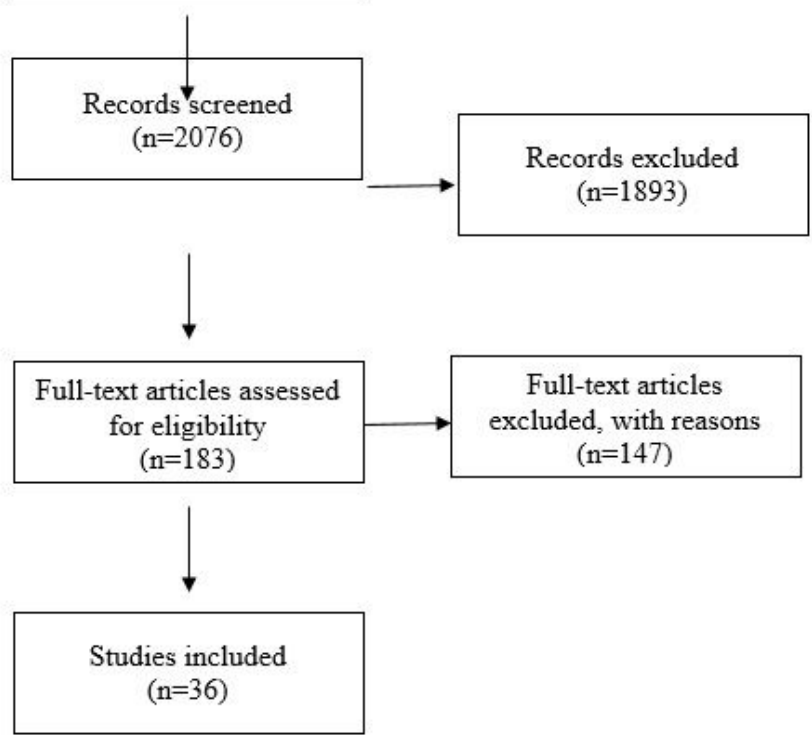

\section{Figure 1}

Flowchart of the screening process 